\title{
Comparison Theorem for Nonlinear Path-Dependent Partial Differential Equations
}

\author{
Falei Wang \\ School of Mathematics, Shandong University, Jinan, Shandong 250100, China \\ Correspondence should be addressed to Falei Wang; flwang1987@163.com
}

Received 27 March 2014; Accepted 19 April 2014; Published 29 April 2014

Academic Editor: Tongxing Li

Copyright (C) 2014 Falei Wang. This is an open access article distributed under the Creative Commons Attribution License, which permits unrestricted use, distribution, and reproduction in any medium, provided the original work is properly cited.

We introduce a type of fully nonlinear path-dependent (parabolic) partial differential equation (PDE) in which the path $\omega_{t}$ on an interval $[0, t]$ becomes the basic variable in the place of classical variables $(t, x) \in[0, T] \times \mathbb{R}^{d}$. Then we study the comparison theorem of fully nonlinear PPDE and give some of its applications.

\section{Introduction}

Motivated by uncertainty problems, risk measures, and the superhedging in finance mathematics, Peng [1] systemically established $G$-expectation theory. In the $G$-expectation framework, the notion of $G$-Brownian motion and the corresponding stochastic calculus of Itô's type were established. The key issue is that $G$-diffusion processes are connected to a large class of fully nonlinear PDEs in the Markov case studied by Peng [2] and Soner et al. [3].

Recently, Dupire [4] and Cont and Fournié [5] introduced a new functional Itô formula which nontrivially generalized the classical one through new notion-path derivatives (see $[6,7]$ for more general and systematic research). It extends the Itô stochastic calculus to functionals of a given process. It provides an excellent tool for the study of path-dependence. In fact, they showed that a smooth path functional solves a linear path-dependent PDE if its composition with a Brownian motion generates a martingale, which provided a functional extension of the classical Feynman-Kac formula. Moreover, by virtue of a backward stochastic differential equation approach, we can obtain the uniqueness of the smooth solution to the semilinear path-dependent PDE (see also [8-10] and the references therein). However, these methods are mainly based on stochastic calculus.

The objective of this paper is to study fully nonlinear PPDE. We refer to Krylov [11] and Wang [12] for the classical fully nonlinear PDE (see also [13-15]). Peng [16] introduced an approach of frozenness of the main course of the paths where the maximization takes place. This approach is based on techniques of PDE and can be directly applied to treat fully nonlinear path-dependent PDE. The advantage of this PDE approach is that one can treat the solution locally (path by path), whereas stochastic calculus is mainly a global approach. In this paper, we will use this method to obtain a comparison principle of fully nonlinear PPDE. In particular, some properties of the solution to fully nonlinear PPDE are also obtained. We claim that these ideas carry over to much more general frameworks, such as the case of viscosity solution to PPDE. Moreover, this method can have direct applications to stochastic analysis, for example, martingales under a fully nonlinear expectation, stochastic optimal control problems, stochastic games, nonlinear pricing and risk measuring, and backward stochastic differential equations. These more technical details are left to future work and will be presented in forthcoming papers.

The paper is organized as follows. In Section 2, we present some existing results in the theory of Dupire's path derivatives that we will use in this paper. In Section 3, we obtain the comparison theorem of fully nonlinear PPDE and give some of its applications.

\section{Preliminaries}

In this section, we give an overview of the definitions concerning path derivatives. The following notations are mainly from Dupire [4] and Cont and Fournié [5]. 
Let $T>0$ be fixed. For each $t \in[0, T]$, we denote by $\Lambda_{t}$ the set of right continuous $\mathbb{R}^{d}$-valued functions on $[0, t]$. For each $\omega_{T} \in \Lambda_{T}$ the value of $\omega_{T}$ at time $s \in[0, T]$ is denoted by $\omega(s)$. Thus $\omega_{T}=\omega(s)_{0<s \leq T}$ is a right continuous process on $[0, T]$ and its value at time $s$ is $\omega(s)$. The path of $\omega_{T}$ up to time $t$ is denoted by $\omega_{t}$; that is, $\omega_{t}=\omega(s)_{0 \leq s \leq t} \in \Lambda_{t}$. Denote $\Lambda=\bigcup_{t \in[0, T]} \Lambda_{t}$. We sometimes specifically write

$$
\omega_{t}=\omega(s)_{0 \leq s<t}=\left(\omega(s)_{0 \leq s<t}, \omega(t)\right)
$$

to indicate the terminal position $\omega(t)$ of $\omega_{t}$ which often plays a special role in this framework. For each $\omega_{t} \in \Lambda$ and $x \in$ $\mathbb{R}^{d}$, denote by $\omega(s)$ the value of $\omega_{t}$ at $s \in[0, t]$ and $\omega_{t}^{x}:=$ $\left(\omega(s)_{0 \leq s<t}, \omega(t)+x\right)$ which is also an element of $\Lambda_{t}$.

Now consider the function $u$ of path; that is, $u: \Lambda \mapsto \mathbb{R}$. This function $u=u\left(\omega_{t}\right)_{\omega_{t} \in \Lambda}$ can be also regarded as a family of real valued functions:

$$
\begin{array}{r}
u\left(\omega_{t}\right)=u\left(t, \omega_{t}(s)_{0 \leq s \leq t}\right)=u\left(t, \omega(s)_{0 \leq s<t}, \omega(t)\right), \\
\omega_{t} \in \Lambda_{t}, \quad t \in[0, T] .
\end{array}
$$

Denote $u\left(\omega_{t}^{x}\right):=u\left(t, \omega(s)_{0 \leq s<t}, \omega(t)+x\right)$, for $\omega_{t} \in \Lambda_{t}, x \in \mathbb{R}^{d}$.

We introduce the distance on $\Lambda$. Let $\langle\cdot, \cdot\rangle$ and $|\cdot|$ denote the inner product and norm in $\mathbb{R}^{d}$. For each $0 \leq t \leq \bar{t} \leq T$ and $\omega_{t}, \bar{\omega}_{\bar{t}} \in \Lambda$, denote

$$
\begin{gathered}
\left\|\omega_{t}\right\|:=\sup _{s \in[0, t]}\left|\omega_{t}(s)\right|, \\
d_{\infty}\left(\omega_{t}, \bar{\omega}_{\bar{t}}\right):=\sup _{s \in[0, t \vee \bar{t}]}|\omega(s \wedge t)-\bar{\omega}(s \wedge \bar{t})|+|t-\bar{t}|^{1 / 2} .
\end{gathered}
$$

It is obvious that $\Lambda_{t}$ is a Banach space with respect to $\|\cdot\|$. Since $\Lambda$ is not a linear space, $d_{\infty}$ is not a norm.

Definition 1 (continuous). A function $u: \Lambda \mapsto \mathbb{R}$ is said to be $\Lambda$-continuous at $\omega_{t} \in \Lambda$, if for any $\varepsilon>0$ there exists $\delta>$ 0 such that for each $\bar{\omega}_{\bar{t}} \in \Lambda$ with $d_{\infty}\left(\omega_{t}, \bar{\omega}_{\bar{t}}\right)<\delta$ we have $\left|u\left(\omega_{t}\right)-u\left(\bar{\omega}_{\bar{t}}\right)\right|<\varepsilon . u$ is said to be $\Lambda$-continuous if it is $\Lambda$ continuous at each $\omega_{t} \in \Lambda$.

Remark 2. In our framework we often regard $u\left(\omega_{t}^{x}\right)$ as a function of $t, \omega_{t}$, and $x$; that is, $u\left(\omega_{t}^{x}\right)=u\left(t, \omega(s)_{0 \leq s<t}, \omega(t)+\right.$ $x)$. Thus, for a fixed $\omega_{t} \in \Lambda, u\left(\omega_{t}^{x}\right)$ is regarded as a function of $(t, x) \in[0, T] \times \mathbb{R}^{d}$.

Definition 3. Given $u: \Lambda \mapsto \mathbb{R}$ and $\omega_{t} \in \Lambda$, if there exists $p \in \mathbb{R}^{d}$, such that

$$
u\left(\omega_{t}^{x}\right)=u\left(\omega_{t}\right)+\langle p, x\rangle+o(|x|) \quad \text { as } x \longrightarrow 0, x \in \mathbb{R}^{d},
$$

then we say that $u$ is (vertically) differentiable at $\omega_{t}$ and denote $D_{x} u\left(\omega_{t}\right)=p \cdot u$ is said to be vertically differentiable in $\Lambda$ if $D_{x} u\left(\omega_{t}\right)$ exists for each $\omega_{t} \in \Lambda$. We can similarly define the Hessian $D_{x x} u\left(\omega_{t}\right)$. It is an $\mathbb{S}(d)$-valued function defined on $\Lambda$, where $\mathbb{S}(d)$ is the space of all $d \times d$ symmetric matrices.
For each $\omega_{t} \in \Lambda, \bar{\omega}_{\bar{t}} \in \Lambda$, with $\bar{t} \geq t$, set

$$
\begin{array}{r}
\omega_{t, \bar{t}}(r):=\omega(r) \mathbf{1}_{[0, t)}(r)+\omega(t) \mathbf{1}_{[t, \bar{t}]}(r), \quad r \in[0, \bar{t}], \\
\omega_{t} \otimes \bar{\omega}_{\bar{t}}(r):=\omega(r) \mathbf{1}_{[0, t)}(r)+(\bar{\omega}(r)-\bar{\omega}(t)+\omega(t)) \mathbf{1}_{[t, \overline{\bar{t}}]}(r), \\
r \in[0, \bar{t}] .
\end{array}
$$

It is clear that $\omega_{t, \bar{t}} \in \Lambda_{\bar{t}}$ and $\omega_{t} \otimes \bar{\omega}_{\bar{t}} \in \Lambda_{\bar{t}}$.

Definition 4. For a given $\omega_{t} \in \Lambda$ if

$$
u\left(\omega_{t, s}\right)=u\left(\omega_{t}\right)+a(s-t)+o(|s-t|) \quad \text { as } s \longrightarrow t, s \geq t,
$$

then we say that $u\left(\omega_{t}\right)$ is (horizontally) differentiable in $t$ at $\omega_{t}$ and denote $D_{t} u\left(\omega_{t}\right)=a . u$ is said to be horizontally differentiable in $\Lambda$ if $D_{t} u\left(\omega_{t}\right)$ exists for each $\omega_{t} \in \Lambda$.

Definition 5. Define $\mathbb{C}^{j, k}(\Lambda)$ as the set of functions $u$ defined on $\Lambda$ which are $j$ times horizontally and $k$ times vertically differentiable in $\Lambda$, such that all these derivatives are $\Lambda$ continuous.

Example 6. If $u\left(\omega_{t}\right)=f(t, \omega(t))$ with $f \in C^{1,1}([0, T) \times \mathbb{R})$, then

$$
D_{t} u\left(\omega_{t}\right)=\partial_{t} f(t, \omega(t)), \quad D_{x} u\left(\omega_{t}\right)=\partial_{x} f(t, \omega(t)),
$$

which is the classic derivative. In general, these derivatives also satisfy the classic properties: linearity, product, and chain rule.

\section{Comparison Theorem for Fully Nonlinear PPDE}

Now we introduce the following fully nonlinear pathdependent PDE:

$$
\begin{array}{r}
D_{t} u\left(\omega_{t}\right)+G\left(\omega_{t}, u\left(\omega_{t}\right), D_{x} u\left(\omega_{t}\right), D_{x x}^{2} u\left(\omega_{t}\right)\right)=0, \\
\omega_{t} \in \Lambda, \\
u\left(\omega_{T}\right)=\Phi\left(\omega_{T}\right), \quad \omega_{T} \in \Lambda_{T},
\end{array}
$$

where $u: \Lambda \mapsto \mathbb{R}$ and $G: \Lambda \times \mathbb{R} \times \mathbb{R}^{d} \times \mathbb{S}(d) \mapsto \mathbb{R}$ are continuous functions. Moreover, $G$ satisfies the following elliptic conditions: for each $\omega_{t} \in \Lambda, r \in \mathbb{R}, p \in \mathbb{R}^{d}, X, Y \in$ $\mathbb{S}(d)$

$$
G\left(\omega_{t}, r, p, X\right) \geq G\left(\omega_{t}, r, p, Y\right), \quad \text { whenever } X \geq Y
$$

and, for each $r_{1}, r_{2} \in \mathbb{R}, p_{1}, p_{2} \in \mathbb{R}^{d}, X_{1}, X_{2} \in \mathbb{S}(d)$, there exists some constant $C$ such that

$$
\begin{aligned}
& \left|G\left(\omega_{t}, r_{1}, p_{1}, X_{1}\right)-G\left(\omega_{t}, r_{2}, p_{2}, X_{2}\right)\right| \\
& \quad \leq C\left(\left|r_{1}-r_{2}\right|+\left|p_{1}-p_{2}\right|+\left|X_{1}-X_{2}\right|\right) .
\end{aligned}
$$


Definition 7. A function $u \in \mathbb{C}^{1,2}(\Lambda)$ is called a $\mathbb{C}^{1,2}$-solution of the path-dependent $\operatorname{PDE}(8)$ if, for each $\omega_{t} \in \Lambda, t \in$ $[0, T)$, equality $(8)$ is satisfied. $u$ is called a subsolution (resp., supersolution) of (8) if the "=" in (8) is replaced by " $\geq$ " (resp., “ $\leq$ ").

Remark 8. The solution of classical PDE is a special case when $u\left(\omega_{t}\right)=\bar{u}(t, \omega(t)), \bar{u} \in C^{1,2}([0, T) \times \mathbb{R})$. Indeed, for each $\omega_{t} \in$ $\Lambda$ and $t \in[0, T)$,

$$
\begin{gathered}
\partial_{t} \bar{u}(t, \omega(t))=D_{t} u\left(\omega_{t}\right), \\
\partial_{x} \bar{u}(t, \omega(t))=D_{x} u\left(\omega_{t}\right), \\
\partial_{x x}^{2} \bar{u}(t, \omega(t))=D_{x x}^{2} u\left(\omega_{t}\right),
\end{gathered}
$$

and thus $\bar{u}(t, x)$ is a classical solution of PDE.

The following result is the so-called comparison principle or comparison theorem of path-dependent PDE.

Theorem 9. Suppose $u_{1}$ is a $\mathbb{C}^{1,2}$-subsolution and $u_{2}$ is a $\mathbb{C}^{1,2}$ supersolution. Moreover, $u_{1}$ is bounded from above and $u_{2}$ is bounded from below. Then the maximum principle holds: if $u_{1}\left(\omega_{T}\right) \leq u_{2}\left(\omega_{T}\right)$ for all $\omega_{T} \in \Lambda_{T}$, then $u_{1}\left(\omega_{t}\right) \leq u_{2}\left(\omega_{t}\right)$ for each $\omega_{t} \in \Lambda$.

Remark 10. In the case when $G\left(\omega_{t}, r, p, X\right)=(1 / 2) \operatorname{tr}[X]+$ $f\left(\omega_{t}, r, p\right)$ for some Lipschitz function $f$ on $\Lambda \times \mathbb{R} \times \mathbb{R}^{d}$, the above result is the comparison theorem of semilinear pathdependent PDE, which is given by [9].

In order to prove Theorem 9, we will make use of the following definitions.

Definition 11. $u: \Lambda \mapsto R$ is said to be in $\operatorname{USC}_{*}(\Lambda)$, if it satisfies the following conditions:

(i) for each fixed $\omega_{\hat{t}} \in \Lambda, \bar{u}(t, x):=u\left(\left(\omega_{\hat{t}}^{x}\right)_{\hat{t}, t-\hat{t}}\right)$ is an upper semicontinuous function of $(t, x) \in[\widehat{t}, T] \times \mathbb{R}^{d}$,

(ii) for each $\omega_{t} \in \Lambda$ with $t_{i} \uparrow t, \limsup _{i \rightarrow \infty} u\left(\omega_{t_{i}}\right) \leq$ $\sup _{x} u\left(\omega_{t}^{x}\right)$.

We also denote $\operatorname{LSC}_{*}(\Lambda):=\left\{-u \mid u \in \operatorname{USC}_{*}(\Lambda)\right\}$. A function $u \in \operatorname{USC}_{*}(\Lambda)$ (resp., $u \in \operatorname{LSC}_{*}(\Lambda)$ ) is called a $\mathscr{C}$-upper (resp., $\mathscr{C}$-lower) semicontinuous function. $u \in$ $\mathscr{C}(\Lambda):=\operatorname{USC}_{*}(\Lambda) \cap \operatorname{LSC}_{*}(\Lambda)$ is called a $\mathscr{C}$-continuous function.

Definition 12. Define $\mathscr{C}^{1,2}(\Lambda)$ as the set of functions $u \in$ $\mathscr{C}(\Lambda) \cup \mathbb{C}(\Lambda)$, such that, for each $\omega_{t} \in \Lambda, D_{t} u\left(\omega_{t}\right), D_{x} u\left(\omega_{t}\right)$, $D_{x x} u\left(\omega_{t}\right)$ exist.

Definition 13. A function $u \in \mathscr{C}^{1,2}(\Lambda)$ is called a $\mathscr{C}^{1,2}$-solution of the path-dependent PDE (8) if for each $\omega_{t} \in \Lambda, t \in[0, T)$ equality (8) is satisfied. $u$ is called a $\mathscr{C}^{1,2}$-subsolution (resp., $\mathscr{C}^{1,2}$-supersolution) of $(8)$ if the "=" in $(8)$ is replaced by " $\geq$ " (resp., “ $\leq ")$.

Theorem 14. Suppose $u_{1}$ is a $\mathscr{C}^{1,2}$-subsolution of PPDE (8) with $G_{1}$ and $u_{2}$ is a $\mathscr{C}^{1,2}$-supersolution of PPDE (8) with $G_{2}$, where $G_{1}\left(\omega_{t}, r, p, X\right) \leq G_{2}\left(\omega_{t}, r, p, X\right)$ for each $\left(\omega_{t}, r, p, X\right) \in$ $\Lambda \times \mathbb{R} \times \mathbb{R}^{d} \times \mathbb{S}(d)$. Moreover, $u_{1}$ is bounded from above and $u_{2}$ is bounded from below. Then the maximum principle holds: if $u_{1}\left(\omega_{T}\right) \leq u_{2}\left(\omega_{T}\right)$ for all $\omega_{T} \in \Lambda_{T}$, then $u_{1}\left(\omega_{t}\right) \leq u_{2}\left(\omega_{t}\right)$ for each $\omega_{t} \in \Lambda$.

It is obvious that $\mathbb{C}^{1,2}(\Lambda) \subset \mathscr{C}^{1,2}(\Lambda)$. Then Theorem 9 is a direct consequence of Theorem 14 .

For each $\alpha>0$ and $\omega_{t} \in \Lambda$, set

$$
w_{\alpha}\left(\omega_{t}\right)=u_{1}\left(\omega_{t}\right)-u_{2}\left(\omega_{t}\right)-\alpha \omega^{2}(t) .
$$

In order to prove Theorem 14, we need the following lemma, which is essentially from Peng [16, Lemma 6].

Lemma 15. If $w_{\alpha}\left(\omega_{t}\right)>0$ for some $\omega_{t} \in \Lambda$, then there exists $\bar{\omega}_{\bar{t}} \in \Lambda$, satisfying $\bar{t} \geq t, w_{\alpha}\left(\omega_{t}\right) \leq w_{\alpha}\left(\bar{\omega}_{\bar{t}}\right)$, and $\bar{\omega}_{\bar{t}}=\omega_{t} \otimes \bar{\omega}_{\bar{t}}$, such that

$$
w_{\alpha}\left(\bar{\omega}_{\bar{t}}\right)=\sup _{\gamma_{t} \in \Lambda, t \geq \bar{t}} w_{\alpha}\left(\bar{\omega}_{\bar{t}} \otimes \gamma_{t}\right)
$$

Proof. For each $\omega_{t} \in \Lambda$ and $w_{\alpha}\left(\omega_{t}\right)>0$, we can find $\hat{x} \in \mathbb{R}$ such that

$$
w_{\alpha}\left(\omega_{t}^{\widehat{x}}\right)=\sup _{x} w_{\alpha}\left(\omega_{t}^{x}\right),
$$

and for each $\omega_{t} \in \Lambda$ with $t_{i} \uparrow t$ we get

$$
\limsup _{i \rightarrow \infty} w_{\alpha}\left(\omega_{t_{i}}\right) \leqslant \sup _{x} w_{\alpha}\left(\omega_{t}^{x}\right) .
$$
[16].

Then the proof is immediate in light of Lemma 6 of Peng

Lemma 16. Let $u \in \mathscr{C}^{1,2}(\Lambda)$ and $\bar{\omega}_{\bar{t}} \in \Lambda$ be given satisfying $u\left(\bar{\omega}_{\bar{t}}\right) \geq u\left(\bar{\omega}_{\bar{t}} \otimes \omega_{t}\right)$ for all $\omega_{t} \in \Lambda, t \geq \bar{t}$. Then

$$
D_{t} u\left(\bar{\omega}_{\bar{t}}\right) \leq 0, \quad D_{x} u\left(\bar{\omega}_{\bar{t}}\right)=0, \quad D_{x x}^{2} u\left(\bar{\omega}_{\bar{t}}\right) \leq 0 .
$$

Proof. Since $u\left(\bar{\omega}_{\bar{t}}\right) \geq u\left(\bar{\omega}_{\bar{t}, \bar{t}+\delta}\right)$ for each $\delta$, we conclude

$$
D_{t} u\left(\bar{\omega}_{\bar{t}}\right) \leq 0 .
$$

For each $x \in \mathbb{R}^{d}$,

$$
u\left(\bar{\omega}_{\bar{t}}\right)=\sup _{x} u\left(\bar{\omega}_{\bar{t}}^{x}\right)
$$

and thus

$$
D_{x} u\left(\bar{\omega}_{\bar{t}}\right)=0, \quad D_{x x}^{2} u\left(\bar{\omega}_{\bar{t}}\right) \leq 0,
$$

which is the desired result.

Now we are going to give the proof of Theorem 14.

Proof of Theorem 14. We first observe that, for $\delta>0$, the functions defined by $\bar{u}_{1}:=u_{1}-\delta / t$ are a subsolution of

$$
\begin{array}{r}
D_{t} \bar{u}_{1}\left(\omega_{t}\right)+\bar{G}_{1}\left(\omega_{t}, \bar{u}_{1}\left(\omega_{t}\right), D_{x} \bar{u}_{1}\left(\omega_{t}\right),\right. \\
\left.D_{x x} \bar{u}_{1}\left(\omega_{t}\right)\right)=\frac{\delta}{t^{2}},
\end{array}
$$


where $\bar{G}_{1}\left(\omega_{t}, r, p, X\right):=G_{1}\left(\omega_{t}, r+\delta / t, p, X\right)$. It is easy to check that the function $\bar{G}_{1}$ satisfies the same conditions as $G_{1}$. Since $u_{1} \leq u_{2}$ follows from $\bar{u}_{1} \leq u_{2}$ in the limit $\delta \rightarrow 0$, it suffices to prove Theorem 14 under the additional assumption

$$
\begin{gathered}
D_{t} u_{1}\left(\omega_{t}\right)+G_{1}\left(\omega_{t}, u_{1}\left(\omega_{t}\right), D_{x} u_{1}\left(\omega_{t}\right), D_{x x} u_{1}\left(\omega_{t}\right)\right) \geq c, \\
c=\frac{\delta}{T^{2}}, \\
\lim _{t \rightarrow 0} u\left(\omega_{t}\right)=-\infty, \quad \text { uniformly on }[0, T) .
\end{gathered}
$$

We make the following assumption.

(A) For each $\omega_{t} \in \Lambda, u, v \in \mathbb{R}, p \in \mathbb{R}^{d}$, and $Q \in \mathbb{S}(d)$ such that $u \geq v$, we have

$$
G\left(\omega_{t}, u, p, Q\right) \leq G\left(\omega_{t}, v, p, Q\right) .
$$

Set $\bar{u}_{1}\left(\omega_{t}\right)=u_{1}\left(\omega_{t}\right) e^{-\lambda t}, \bar{u}_{2}\left(\omega_{t}\right)=u_{2}\left(\omega_{t}\right) e^{-\lambda t}$, for some $\lambda<$ $-C$. Then it is easy to check that $\bar{u}_{i}(i=1,2)$ is a subsolution or supersolution of

$$
D_{t} \bar{u}_{i}\left(\omega_{t}\right)+\bar{G}_{i}\left(\omega_{t}, \bar{u}_{i}\left(\omega_{t}\right), D_{x} \bar{u}_{i}\left(\omega_{t}\right), D_{x x} \bar{u}_{i}\left(\omega_{t}\right)\right)=0,
$$

where, for each $\left(\omega_{t}, u, p, Q\right) \in \Lambda \times \mathbb{R} \times \mathbb{R}^{d} \times \mathbb{S}(d)$, $\bar{G}_{i}\left(\omega_{t}, r, p, Q\right)$ is given by

$$
\bar{G}_{i}\left(\omega_{t}, u, p, Q\right)=\lambda u+e^{-\lambda t} G_{i}\left(\omega_{t}, e^{\lambda t} u, e^{\lambda t} p, e^{\lambda t} Q\right),
$$

which satisfies the assumption (A). Since $\bar{u}_{1} \leq \bar{u}_{2}$ implies $u_{1} \leq u_{2}$, it suffices to prove Theorem 14 under the additional assumption (A).

Without loss of generality, assume $w_{\alpha} \leq C^{2}$. Suppose by the contrary that there exist $\omega_{t_{0}}^{0} \in \Lambda, t_{0}<T$, and $\sqrt{\alpha}<$ $c / 4\left(C \vee C^{2}\right) \wedge 1$, such that

$$
m_{0}:=w_{\alpha}\left(\omega_{t_{0}}^{0}\right)>0 .
$$

Then, by Lemma 15 , there exists $\bar{\omega}_{\bar{t}} \in \Lambda$ such that

$$
w_{\alpha}\left(\bar{\omega}_{\bar{t}}\right)=\sup _{\gamma_{t} \in \Lambda, t \geq \bar{t}} w_{\alpha}\left(\bar{\omega}_{\bar{t}} \otimes \gamma_{t}\right) \geq m_{0} .
$$

Consequently,

$$
\begin{gathered}
D_{t} u_{1}\left(\bar{\omega}_{\bar{t}}\right)-D_{t} u_{2}\left(\bar{\omega}_{\bar{t}}\right) \leq 0, \\
D_{x} u_{1}\left(\bar{\omega}_{\bar{t}}\right)-D_{x} u_{2}\left(\bar{\omega}_{\bar{t}}\right)=2 \alpha \bar{\omega}(\bar{t}), \\
D_{x x} u_{1}\left(\bar{\omega}_{\bar{t}}\right)-D_{x x} u_{2}\left(\bar{\omega}_{\bar{t}}\right) \leq 2 \alpha .
\end{gathered}
$$

Since $\alpha \bar{\omega}^{2}(\bar{t}) \leq C^{2}$, we have $\alpha \bar{\omega}(\bar{t}) \leq C \sqrt{\alpha}$. Then

$$
\begin{aligned}
& 0<c \\
& \leq D_{t} u_{1}\left(\bar{\omega}_{\bar{t}}\right)+G_{1}\left(\bar{\omega}_{\bar{t}}, u_{1}\left(\bar{\omega}_{\bar{t}}\right), D_{x} u_{1}\left(\bar{\omega}_{\bar{t}}\right), D_{x x} u_{1}\left(\bar{\omega}_{\bar{t}}\right)\right) \\
& \leq D_{t} u_{2}\left(\bar{\omega}_{\bar{t}}\right)+G_{2}\left(\bar{\omega}_{\bar{t}}, u_{2}\left(\bar{\omega}_{\bar{t}}\right), D_{x} u_{2}\left(\bar{\omega}_{\bar{t}}\right)+2 \alpha \bar{\omega}(\bar{t}),\right. \\
& \left.\quad D_{x x} u_{2}\left(\bar{\omega}_{\bar{t}}\right)+2 \alpha\right) \\
& \leq 2 C(\alpha+C \sqrt{\alpha})<c .
\end{aligned}
$$

This induces a contradiction and the proof is completed.
Corollary 17. The path-dependent PDE (8) has at most one bounded $\mathbb{C}^{1,2}$-solution.

Lemma 18. If $u$ is a bounded $\mathscr{C}^{1,2}$-subsolution of PPDE (8) and $\Phi$ is a bounded function on $\Lambda_{T}$, then, for each $\omega_{t} \in \Lambda$,

$$
u\left(\omega_{t}\right) \leq \sup _{\omega_{T} \in \Lambda_{T}} \Phi\left(\omega_{T}\right)
$$

Proof. Since $\sup _{\omega_{T} \in \Lambda_{T}}\left|\Phi\left(\omega_{T}\right)\right|$ is a $\mathscr{C}^{1,2}$-supersolution of PPDE (8), applying Theorem 14, we have the desired result.

Remark 19. If $u\left(\omega_{t}\right)=\bar{u}(t, \omega(t))$ is the $\mathbb{C}^{1,2}$-solution of PPDE (8) for some bounded $\bar{u} \in C^{1,2}([0, T) \times \mathbb{R})$, then the above theorem is the classical comparison theorem of PDE of [17].

Example 20. Consider the following PPDE:

$$
\begin{gathered}
D_{t} u\left(\omega_{t}\right)+G\left(D_{x x}^{2} u\left(\omega_{t}\right)\right)=0, \quad \omega_{t} \in \Lambda, \\
u\left(\omega_{T}\right)=\Phi\left(\omega_{T}\right), \quad \omega \in \Lambda_{T},
\end{gathered}
$$

where $G(a):=(1 / 2)\left(\bar{\sigma}^{2} a^{+}-\underline{\sigma}^{2} a^{-}\right)$for some $0<\underline{\sigma}^{2} \leq \bar{\sigma}^{2}<\infty$.

If $\Phi(\omega)=\varphi(\omega(t), \omega(\bar{T}))$ for some bounded Lipschitz function $\varphi \in C\left(\mathbb{R}^{2}\right)$ and $t \in[0, T]$, then we can solve the PPDE (30) by the following method.

First consider the following system of fully nonlinear parabolic partial differential equations, defined on $[0, T] \times \mathbb{R}^{2}$ and parameterized by $y \in \mathbb{R}$ :

$$
\begin{gathered}
\partial_{s} v_{1}(s, x, y)+G\left(\partial_{x x}^{2} v_{1}(s, x, y)\right)=0 \\
v_{1}(T, x, y)=\varphi(x, y)
\end{gathered}
$$

and, then, another one defined on $[0, t] \times \mathbb{R}$ :

$$
\begin{gathered}
\partial_{s} v_{2}(s, x)+G\left(\partial_{x x}^{2} v_{2}(s, x)\right)=0, \\
v_{2}(t, x)=v_{1}(t, x, x) .
\end{gathered}
$$

From Krylov [11] and Wang [18], for each $y \in \mathbb{R}, v_{1}(s, x, y) \in$ $C^{1+\alpha / 2,2+\alpha}([0, T-\kappa) \times \mathbb{R})$, and $v_{2}(s, x) \in C^{1+\alpha / 2,2+\alpha}([0, t-\kappa) \times$ $\mathbb{R})$, where $\alpha \in(0,1)$ and $\kappa>0$.

Denote $u\left(\omega_{s}\right):=v_{1}(s, \omega(s), \omega(t)) 1_{t \leq s}+v_{2}(s, \omega(s)) 1_{s<t}$; we obtain $u \in \mathscr{C}^{1,2}(\Lambda)$. Applying Theorem $14, u$ is the unique $\mathscr{C}^{1,2}$-solution of PPDE (30). Indeed, $u\left(\omega_{t}\right)$ is the conditional $G$-expectation of $\Phi\left(\omega_{T}\right)$ (see $\left.[19,20]\right)$.

\section{Conflict of Interests}

The author declares that there is no conflict of interests regarding the publication of this paper.

\section{Acknowledgment}

This work is supported by the Graduate Independent Innovation Foundation (YZC12062) of Shandong University. 


\section{References}

[1] S. Peng, "Multi-dimensional G-Brownian motion and related stochastic calculus under G-expectation," Stochastic Processes and Their Applications, vol. 118, no. 12, pp. 2223-2253, 2008.

[2] S. Peng, "Survey on normal distributions, central limit theorem, Brownian motion and the related stochastic calculus under sublinear expectations," Science in China A: Mathematics, vol. 52, no. 7, pp. 1391-1411, 2009.

[3] H. M. Soner, N. Touzi, and J. Zhang, "Wellposedness of second order backward SDEs," Probability Theory and Related Fields, vol. 153, no. 1-2, pp. 149-190, 2012.

[4] B. Dupire, "Functional Itô calculus," Portfolio Research Paper 2009-04, Bloomberg, 2009.

[5] R. Cont and D.-A. Fournié, "A functional extension of the Itô formula," Comptes Rendus Mathematique, vol. 348, no. 1-2, pp. 57-61, 2010.

[6] R. Cont and D.-A. Fournié, "Change of variable formulas for non-anticipative functionals on path space," Journal of Functional Analysis, vol. 259, no. 4, pp. 1043-1072, 2010.

[7] R. Cont and D.-A. Fournié, "Functional Itô calculus and stochastic integral representation of martingales," The Annals of Probability, vol. 41, no. 1, pp. 109-133, 2013.

[8] É. Pardoux and S. Peng, "Backward stochastic differential equations and quasilinear parabolic partial differential equations," in Stochastic Partial Differential Equations and Their Applications, vol. 176 of Lecture Notes in Control and Information Sciences, pp. 200-217, Springer, Berlin, Germany, 1992.

[9] I. Ekren, C. Keller, N. Touzi, and J. Zhang, "On viscosity solutions of path dependent PDEs," The Annals of Probability, vol. 42, no. 1, pp. 204-236, 2014.

[10] Y. Xu, "Probabilistic solutions for a class of path-dependent Hamilton-Jacobi-Bellman equations," Stochastic Analysis and Applications, vol. 31, no. 3, pp. 440-459, 2013.

[11] N. V. Krylov, Nonlinear Parabolic and Elliptic Equations of the Second Order, Reidel Publishing Company, 1987, Original Russian version by Nauka, Moscow, 1985.

[12] L. Wang, "On the regularity theory of fully nonlinear parabolic equations: I," Communications on Pure and Applied Mathematics, vol. 45, no. 1, pp. 27-76, 1992.

[13] L. A. Caffarelli and X. Cabre, "Fully nonlinear elliptic partial differential equations," American Mathematical Society, vol. 34, no. 2, pp. 187-191, 1997.

[14] M. G. Crandall and P.-L. Lions, "Viscosity solutions of Hamilton-Jacobi equations," Transactions of the American Mathematical Society, vol. 277, no. 1, pp. 1-42, 1983.

[15] P.-L. Lions, "Optimal control of diffusion processes and hamilton-jacobi-bellman equations part 2 : viscosity solutions and uniqueness," Communications in Partial Differential Equations, vol. 8, no. 11, pp. 1229-1276, 1983.

[16] S. Peng, "Note on viscosity solution of path-dependent PDE and G-martingales lecturenotes," http://xxx.tau.ac.il/abs/1106 $.1144 \mathrm{v} 1$.

[17] M. G. Crandall, H. Ishii, and P.-L. Lions, "User's guide to viscosity solutions of second order partial differential equations," Bulletin of the American Mathematical Society, vol. 27, no. 1, pp. $1-67,1992$.

[18] L. Wang, "On the regularity theory of fully nonlinear parabolic equations: II," Communications on Pure and Applied Mathematics, vol. 45, no. 2, pp. 141-178, 1992.
[19] L. Denis, M. Hu, and S. Peng, "Function spaces and capacity related to a sublinear expectation: application to G-Brownian motion paths," Potential Analysis, vol. 34, no. 2, pp. 139-161, 2011.

[20] S. Peng, "Backward stochastic differential equation, nonlinear expectation and their applications," in Proceedings of the International Congress of Mathematicians, pp. 393-432, Hyderabad, India, 2010. 


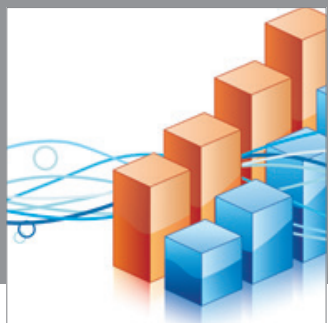

Advances in

Operations Research

mansans

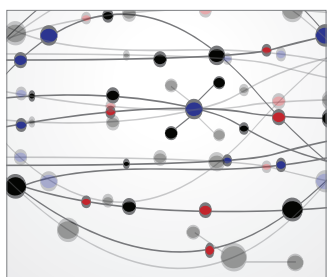

The Scientific World Journal
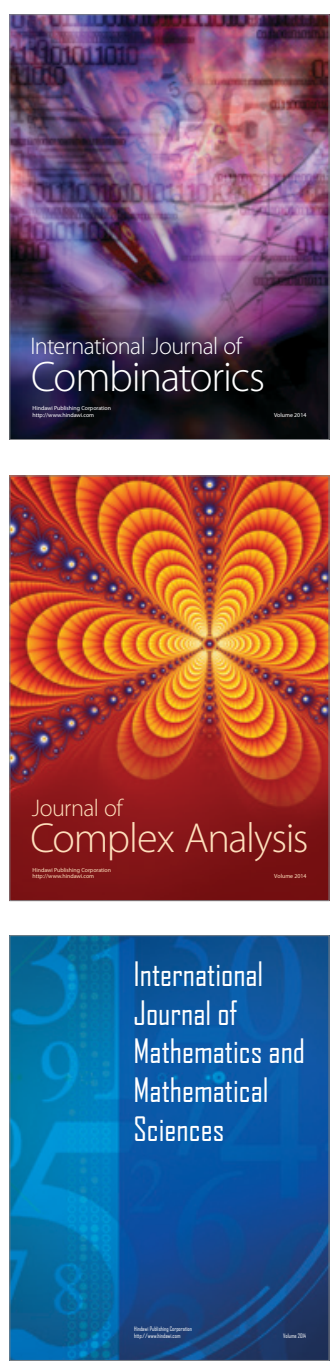
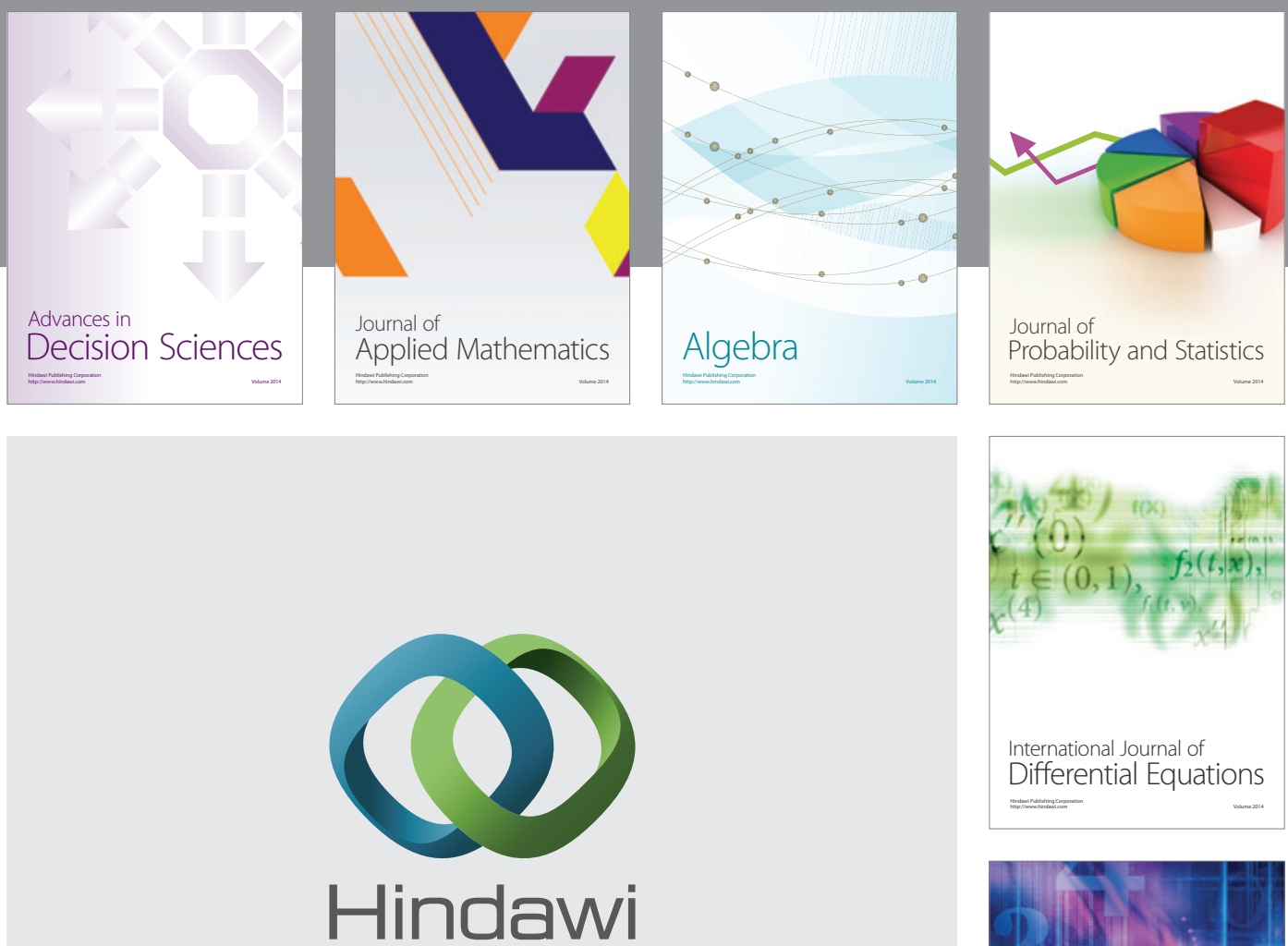

Submit your manuscripts at http://www.hindawi.com
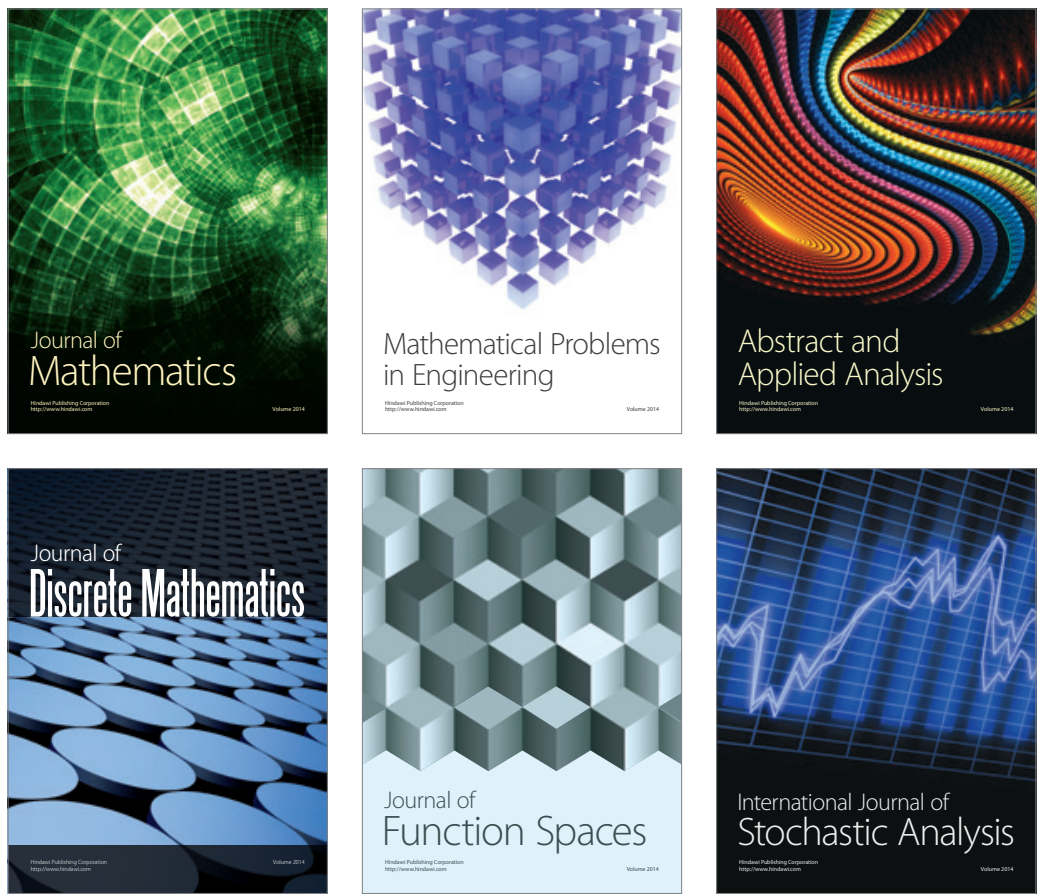

Journal of

Function Spaces

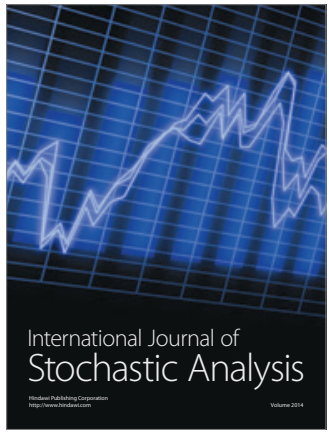

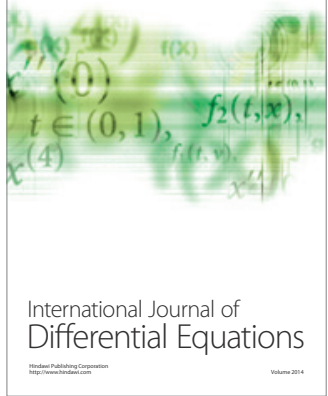
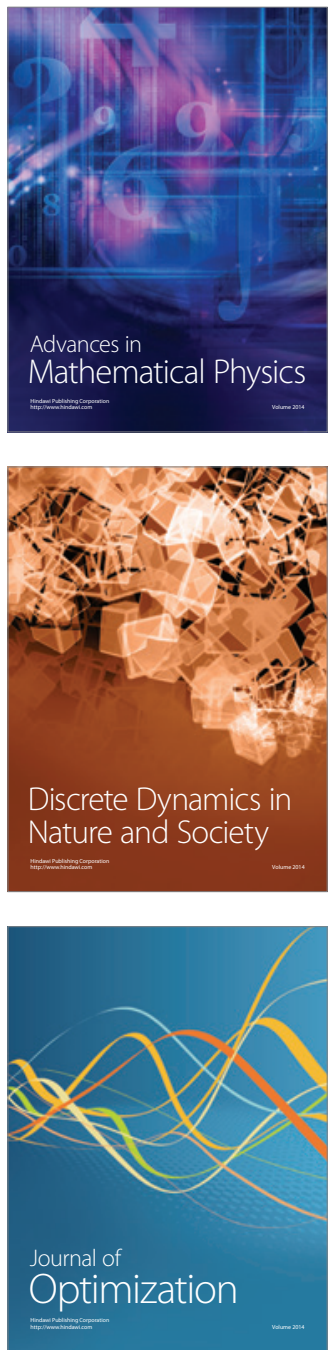